\title{
OPERACIONES DE REDUCCIÓN DE VALENCIA EN EL CRIOLLO INGLÉS DE COSTA RICA
}

\author{
Mario Portilla Chaves
}

\begin{abstract}
RESUMEN
El presente artículo describe seis operaciones de reducción de valencia en el criollo inglés de Costa Rica. Se analizan las construcciones reflexivas, recíprocas, pasivas, medias, con remoción de paciente y con omisión de paciente que existen en esta lengua, desde un punto de vista tipológico-funcional.
\end{abstract}

\section{ABSTRACT}

The present article describes six valence decreasing operations in the English-based Creole of Costa Rica. The reflexive, reciprocal, passive, middle, object demotion, and object omission constructions, that exist in this language are analyzed from a typologicalfunctional point of view.

\section{Introducción}

\subsection{Operaciones de ajuste de valencia}

Desde el punto de vista tipológico-funcional ${ }^{1}$, el término operaciones de ajuste de valencia describe la tipología de estrategias morfosintácticas que son utilizadas en las lenguas del mundo para resolver la falta de congruencia entre las propiedades de la representación conceptual de las cosas y los eventos en el mundo real, y las propiedades de los elementos lingüísticos en las oraciones (Payne 1997: 171), es decir, entre las nociones semántica y gramatical expresadas en el discurso.

La noción semántica de valencia se refiere al número de participantes implicados en una determinada situación expresada por un verbo. Por ejemplo, semánticamente, el verbo golpear refiere una situación que implica necesariamente dos participantes, un agente que ejecuta la acción de golpear y un paciente u objeto que es golpeado. Por su parte, el verbo caminar refiere una situación en la que está implicado solamente un participante: quien realiza el movimiento. Así, se dice que el primer verbo es bivalente, mientras que el segundo es monovalente.

Por otro lado, la noción gramatical de valencia hace referencia al número de argumentos presentes en una determinada cláusula. Estos argumentos se definen como aquellos 
elementos nominales que establecen una relación sintáctica con el verbo en la oración. Así, por ejemplo, según la oración dada, el mismo verbo golpear, que semánticamente es bivalente, puede ser gramaticalmente o bien bivalente como en el caso de decir María golpeó a Juan, o bien monovalente como en el caso de la oración, Juan fue golpeado. En la primera oración, las nociones semánticas de agente y paciente se corresponden con las nociones gramaticales de sujeto y objeto respectivamente. En la segunda oración, la operación morfosintáctica, conocida como voz pasiva, ha reducido la valencia gramatical de un verbo semánticamente bivalente, omitiendo la mención del agente y promoviendo el paciente de la acción a sujeto.

\section{2. Propósito de la investigación}

Las operaciones de ajuste de valencia permiten tanto reducir la valencia gramatical de un verbo como aumentarla. El presente estudio describe las operaciones de reducción de valencia que aparecen en el criollo inglés de Costa Rica de acuerdo con la tipología de operaciones propuesta por Payne (1997: 172).

\section{Construcciones transitivas e intransitivas en el criollo inglés de Costa Rica}

En esta clase de construcciones, existe una congruencia o igualdad entre la valencia semántica del verbo y la valencia gramatical de la cláusula. En las construcciones transitivas, el agente de un verbo de acción se categoriza como sujeto de la cláusula, colocándose a la izquierda del verbo. El paciente de la acción se categoriza, a su vez, como objeto directo de la cláusula y se coloca a la derecha del verbo. Por esto, el criollo inglés de Costa Rica se define como una lengua del tipo SVO.

1. Jan muuv di tíebl.

(Juan mover la mesa)

Juan movió la mesa.

2. Jan kil di láyan.

(Juan matar el león)

Juan mató al león.

3. Di láyan kil Jan.

(El león matar Juan)

El león mató a Juan.

En las construcciones intransitivas, o bien el agente de un movimiento o bien el paciente de un estado o de un proceso aparecen como sujeto de la cláusula, colocándose a la izquierda del verbo. 
4. Jan waak (wan ówa).

(Juan caminar [una hora])

Juan caminó (una hora).

5. Jan di a sliip.

(Juan ANT PRO dormir)

Juan estaba durmiendo.

6. Jan ded (yéside).

(Juan morir [ayer])

Juan murió (ayer).

\section{Operaciones de reducción de valencia que combinan el agente y el paciente}

\subsection{Las construcciones reflexivas}

En este tipo de construcciones, el agente y el paciente implicados en la situación expresada por el verbo se refieren al mismo participante. En el criollo inglés de Costa Rica, hay dos tipos de construcciones reflexivas: una construcción reflexiva léxica y una construcción reflexiva analítica.

En la construcción reflexiva léxica, la forma simple del verbo implica el significado de que el sujeto del verbo es a la vez agente y paciente de la acción expresada por el verbo. Esta construcción es equivalente a una cláusula intransitiva.

7. Jan shiev yéside.

(Juan rasurar ayer)

Juan se rasuró ayer.

8. Jan dres-op.

(Juan vestir-elegantemente)

Juan se vistió elegantemente.

En la construcción reflexiva analítica, el paciente de la acción expresada por el verbo aparece representado, como un objeto directo, por un pronombre reflexivo, el cual es correferencial con el sujeto en persona y número, y por el marcador de reflexividad -self.

9. Jan si im self ina di glaas.

(Juan ver él REF dentro el espejo)

Juan se vió (a sí mismo) en el espejo.

10. Jan an Píita shiev dem self yéside.

(Juan y Pedro rasurar ellos REF ayer)

Juan y Pedro se rasuraron a sí mismos ayer. 
La oración (10) muestra que uno de los usos pragmáticos de la construcción reflexiva analítica es evitar la ambigüedad en la interpretación correcta de la oración como reflexiva, ya que, como se verá más adelante, la sola utilización del verbo simple podría haber dado lugar a una posible interpretación recíproca o incluso pasiva.

A continuación se presenta el paradigma pronominal completo de la construcción reflexiva analítica.

Pronombres reflexivos del criollo inglés de Costa Rica

\begin{tabular}{|c|c|c|c|}
\hline & \multicolumn{2}{|c|}{ Singular } & \multirow[t]{2}{*}{ Plural } \\
\hline & masculino & femenino & \\
\hline $1^{\circ}$ persona & \multirow{2}{*}{\multicolumn{2}{|c|}{$\begin{array}{l}\text { mi self } \\
\text { yu self }\end{array}$}} & wi self $\sim$ ówa self \\
\hline $2^{\circ}$ persona & & & únu self \\
\hline $3^{\circ}$ persona & im self & shi self $\sim$ ar self & dem self \\
\hline
\end{tabular}

\subsection{La construcción recíproca}

Al igual que en el caso de las reflexivas, en la construcción recíproca, el agente de la acción del verbo también participa como paciente de ella, pero de manera recíproca. Como se ha dicho, en esta lengua, la construcción recíproca puede ser expresada léxicamente. Algunos ejemplos de la construcción recíproca léxica son los siguientes:

11. Jan an Mierí márid in Limón.

(Juan y María casar en Limón)

Juan y María se casaron en Limón.

12. Jan an Mierí waz kísin-op.

(Juan y María ANT besar)

Juan y María se estaban besando.

Además, existe en esta lengua la posibilidad de expresar la noción de reciprocidad de la situación de manera analítica, añadiendo la forma iich-ádaz después del verbo. Del mismo modo que en el caso de las reflexivas analíticas, en algunos casos, el uso de la construcción recíproca analítica permite evitar alguna posible ambigüedad en la interpretación correcta de la oración.

13. Jan an Mierí nuo iich-ádaz in Limón.

(Juan y María conocer REC en Limón)

Juan y María se conocieron en Limón. 
14. Jan an Mierí si iich-ádaz ina di glaas.

(Juan y María ver REC en el espejo)

Juan y María se vieron mutuamente en el espejo.

El uso del marcador de reciprocidad evita una interpretación ambigua de la situación expresada en la oración anterior (cf. más adelante la oración 28).

\section{Operaciones de reducción de valencia que degradan el agente}

\subsection{Las construcciones pasivas}

En este tipo de construcciones, normalmente el agente de una acción no aparece expresado en la cláusula, mientras que el paciente es promovido a desempeñar la función gramatical de sujeto del verbo.

Existen dos tipos de pasivas en el criollo inglés de Costa Rica: una léxica y una analítica. En las construcciones pasivas léxicas, el sujeto de la cláusula es el paciente que recibe la acción. El agente de la acción o bien no aparece expresado en la cláusula o bien es categorizado como un complemento, que se introduce por la preposición instrumental bai.

15. Di baal kik.

(La bola patear)

La bola fue pateada.

16. Di láyan kil bai Jan.

(El léon matar por Juan)

El león fue matado por Juan.

Es importante señalar que en la oración (16) la presencia del complemento agente (bai Jan) evita una posible interpretación ambigua en cuanto que especifica quién es el agente de la acción. Eventualmente, la oración Di láyan kil podría haber sido interpretada o bien como una oración pasiva o bien como una intransitiva.

La construcción pasiva analítica se construye por medio del verbo auxiliar get, el cual precede al verbo principal.

17. Jan get shat (bai Mierí).

(Juan AUX disparar [por María])

Juan fue baleado [por María].

18. Di láyan get kil.

(El león AUX matar)

El león fue matado. 
La oración anterior muestra que, a diferencia de la construcción pasiva léxica, la pasiva analítica no es ambigua (cf. Di láyan kil).

\subsection{Las construcciones medias}

$\mathrm{Al}$ igual que en las construcciones pasivas, las construcciones medias promueven a sujeto el paciente de la cláusula. Según Payne (1997: 216), en realidad, estas construcciones se diferencian semánticamente de las pasivas solamente por el hecho de que la situación expresada por el verbo es un proceso que sufre el paciente, en vez de ser una acción.
19. Di glaas brok.
(El vaso quebrar)
El vaso se quebró.

20. Di duor a nak (bikáa a di win).

(La puerta PROG golpear [a-causa de el viento])

La puerta está golpeándose (a causa del viento).

$\mathrm{Al}$ igual que en las pasivas, en estas construcciones, el paciente que sufre el proceso se categoriza formalmente como sujeto de la cláusula. Obviamente, al no tratarse la situación expresada por el verbo de una acción, no existe agente explícito ninguno. Dada la igualdad formal y la gran semejanza semántica entre las construcciones medias y las pasivas léxicas sin complemento agente, puede existir una ambigüedad en la interpretación semántica de una cláusula como (21).

21. Di tíebl muuv.

(La mesa mover)

La mesa se movió (por un temblor). $\quad \Rightarrow$ Interpretación media.

La mesa fue movida (por Juan). $\quad \Rightarrow$ Interpretación pasiva.

\section{Operaciones de reducción de valencia que degradan el paciente}

\subsection{Las construcciones con remoción del paciente}

Esta operación convierte el paciente semántico de un verbo de acción en un complemento circunstancial. De esta forma, las cláusulas transitivas (22a) y (23a), cuyo paciente es categorizado como objeto directo, son semánticamente equivalentes a las cláusulas intransitivas (22b) y (23b) respectivamente, en cuyo caso el paciente aparece como un objeto de preposición.

22a. Jan mount di aas.

(Juan montar el caballo)

$=>$ Cláusula transitiva

Juan montó el caballo. 
22b. Jan mount pan di aas.

(Juan montar en el caballo) $\quad$ => Cláusula intransitiva con remoción de paciente

Juan se montó en el caballo.

23a. Jan uol di kórtin.

(Juan agarrar la cortina) $\quad \Rightarrow$ Cláusula transitiva

Juan agarró la cortina.

23b. Jan uol aan di kórtin.

(Juan agarrar en la cortina) $\quad \Rightarrow$ Cláusula intransitiva con remoción de paciente

Juan se agarró de la cortina.

\subsection{Construcciones con omisión de paciente}

En este tipo de construcciones, el paciente semántico de un verbo de acción es omitido completamente en la cláusula. Al no aparecer ningún objeto directo, la cláusula es intransitiva.

24. Jan yuustu mount.

(Juan HAB montar)

Juan solía montar.

En algunos casos, estas construcciones pueden ser semánticamente ambiguas. La oración (25), por ejemplo, podría ser interpretada, según el contexto, bien como una cláusula intransitiva o bien como una pasiva o como una reflexiva.

25. Jan shat.

(Juan disparar)

Juan le disparó (a un hombre) $\quad \Rightarrow$ Interpretación intransitiva

Juan fue baleado (por un hombre) $\quad \Rightarrow$ Interpretación pasiva

Juan se disparó (a sí mismo) $\quad \Rightarrow$ Interpretación reflexiva

\section{Reducción de la valencia y la transparencia semántica de las lenguas criollas}

La descripción anterior de las operaciones de reducción de valencia pone de manifiesto que, en casi todos los casos, la interpretación semántica del argumento que ocurre a la izquierda del verbo (el sujeto), ya sea como agente, ya sea como paciente, ya sea como agente y paciente a la vez, depende, en realidad, del contexto del discurso.

En otras palabras, no existe ningún indicador formal que permita diferenciar una construcción reflexiva léxica de una recíproca léxica o de una pasiva léxica o de una construcción media o, incluso, de una cláusula intransitiva con omisión de paciente. De hecho, se podría sostener que todas las construcciones mencionadas, en realidad, constituyen el resultado de la aplicación de una única y misma operación formal, la elisión del argumento que no es relevante pragmáticamente. 
Los siguientes ejemplos de oraciones potencialmente ambiguas, al igual que otras mencionadas anteriormente, ilustran el punto anterior.

26. Jan kil.

(Juan matar)

Juan conquistó ( una muchacha). $\quad$ => Interpretación intransitiva

Juan se suicidó.

$\Rightarrow$ Interpretación reflexiva

Juan se mató (en un accidente).

$\Rightarrow$ Interpretación media

Juan fue matado (por alguien).

=> Interpretación pasiva

27. A biit.

(Yo golpear)

Yo gané (en un juego).

=> Interpretación intransitiva

Me pegué a mí mismo (de rabia).

=> Interpretación reflexiva

Me golpeé (involuntariamente).

$\Rightarrow$ Interpretación media

Fui golpeado (por alguien).

=> Interpretación pasiva

28. Jan an Mierí si ina di glaas.

(Juan y María ver en el espejo)

Juan y María vieron (algo) dentro del espejo $\quad \Rightarrow$ Interpretación intransitiva

Juan y María se vieron (mutuamente) en el espejo $\Rightarrow$ Interpretación recíproca

Juan y María se vieron a sí mismos en el espejo $\Rightarrow$ Interpretación reflexiva

Juan y María fueron vistos en el espejo. $\quad \Rightarrow$ Interpretación pasiva

A diferencia de las oraciones (26) y (27), la oración (28) permite también una interpretación recíproca debido a que el sujeto es plural.

Por otro lado, las posibles interpretaciones semánticas del argumento de sujeto como agente o paciente están determinadas también por el hecho de que este sea animado o no. Los ejemplos (29) y (30) muestran que, por tratarse de un ente inanimado, no es posible una interpretación ni intransitiva ni reflexiva de la cláusula, ya que el sujeto sólo puede ser paciente de una acción y nunca agente de ella.

(29) Di miit kyan iit.

(La carne AUX-MOD comer)

La carne puede comerse (porque está suave). $\quad \Rightarrow$ Interpretación media.

La carne puede comerse (porque ya está preparada). $\Rightarrow$ Interpretación pasiva.

(30) Di tíebl muuv.

(La mesa mover)

La mesa se movió (en un temblor). $\quad \Rightarrow$ Interpretación media

La mesa fue movida (por alguien).

$\Rightarrow$ Interpretación pasiva

Como se ha dicho anteriormente, una de las funciones pragmáticas generales de las llamadas construcciones analíticas reflexivas, recíprocas y pasivas es la de evitar posibles ambigüedades en la interpretación semántica de estas cláusulas. En los siguientes ejemplos, solamente la oración (32) es ambigua. 
32. Jan shiev ${ }^{2}$.

(Juan rasurar)

Juan rasura (gente).

Juan se rasuró.

Juan fue rasurado (por alguien).

$=>$ Interpretación intransitiva

$\Rightarrow$ Interpretación reflexiva

$=>$ Interpretación pasiva

33. Jan shiev pípl.

(Juan rasurar gente)

Juan rasura gente. (i.e. Juan es barbero). => Oración transitiva

34. Jan shiev im self.

(Juan rasurar él REF)

Juan se rasuró a sí mismo.

$=>$ Oración reflexiva

35. Jan get shiev.

(Juan AUX rasurar)

Juan fue rasurado.

$=>$ Oración pasiva

Dentro de los estudios criollos, tanto Bickerton $(1981,1984)$ como Seuren \& Wekker (1986) han postulado teorías que sugieren que las lenguas criollas poseen estructuras morfosintácticas que reflejan más directamente algunas estructuras semánticas más básicas. Por esta razón, estos autores y otros más han considerado que la morfosintaxis de las lenguas criollas es, en este sentido, muchas veces más simple que la de otras lenguas. El autor de este trabajo considera que las operaciones de reducción de valencia léxicas descritas anteriormente representan un ejemplo de simplificación de la sintaxis en esta lengua, ya que permiten utilizar pocos recursos morfosintácticos (por ejemplo, marcadores morfológicos, auxiliares, cambios de lugar de argumentos en la cláusula, etc.) para transmitir la información deseada. Esta economía se logra mediante el uso óptimo de la información del contexto (incluyendo la categorización semántica de sintagmas nominales que constituyen los argumentos).

Por ejemplo, como se ha dicho anteriormente, una cláusula que presente como argumento un ente inanimado no podrá ser interpretada nunca como una oración intransitiva ni reflexiva porque semánticamente este argumento nunca podrá ser el agente de una acción, sino solamente el paciente de ella. Tal es el caso de las oraciones (29) y (30) mencionadas arriba, y de la siguiente oración (36) en la cual el elemento nominal, la buena comida, no puede realizar la acción de cocinar, sino sólo recibirla, es decir, ser cocinada.

(36) Di fuud kuk.

(La comida cocinar)

La comida fue cocinada. => Oración pasiva

En este caso, se entiende el concepto de simplificación como el uso más eficiente de las estructuras morfosintácticas de la lengua, cuando se aprovecha la denominada por Seuren y Wekker (1986) transparencia semántica de la relación del argumento presente en la cláusula con el verbo ${ }^{3}$. 


\section{Lista de abreviaturas}

$\begin{array}{lll}\text { ANT } & = & \text { Tiempo anterior } \\ \text { AUX } & = & \text { Verbo auxiliar } \\ \text { AUX-MOD } & = & \text { Verbo auxiliar modal } \\ \text { HAB } & = & \text { Aspecto habitual } \\ \text { PRO } & = & \text { Aspecto progresivo } \\ \text { REC } & = & \text { Partícula anafórica recíproca } \\ \text { REF } & = & \text { Pronombre reflexivo }\end{array}$

\section{Notas}

1. El presente apartado resume fundamentalmente la posición teórica sostenida por Payne (1997: cap. 8).

2. La interpretación del verbo simple como tiempo pasado o no pasado está determinada también por el contexto, cf. Portilla (1996).

3. Una situación de simplificación semejante a la descrita para la sintaxis del criollo inglés de Costa Rica parece ocurrir también en el habla infantil. El siguiente ejemplo tomado de mi hijo a la edad de 3 años ilustra el caso de dos cláusulas formalmente iguales cuyo sujeto es interpretado de manera distinta como agente o paciente de la acción de verbos bivalentes.

(37) Mamá bajar. Perro pegar. $\Rightarrow \quad$ i.e. Mamá, bájeme. Le voy a pegar al perro.

Por el contexto, el argumento de la primera cláusula puede ser interpretado solamente como el agente de la acción (el niño está en brazos de su madre), mientras que el argumento de la segunda cláusula es indudablemente el paciente de la acción descrita por el verbo.

\section{Bibliografía}

Bickerton, Derek. 1981. Roots of language. Ann Arbor: Karoma.

1984. "The language bioprogram hypothesis". The behavioral and brain sciences. 7 : 173-221.

Payne, Thomas. 1997. Describing morphosyntax: a guide for field linguistics. Cambridge: Cambridge University Press.

Portilla, Mario. 1996. "Tiempo, aspecto y modo en el criollo inglés de Costa Rica". Revista de Filología y Lingüística de la Universidad de Costa Rica. 23(1).

Muysken, Pieter \& Norval Smith (eds.). 1986. Substrata versus universal in creole genesis. Amsterdam: Benjamins.

Seuren, P. \& H. Wekker. 1986. "Semantic transparency as a factor in creole genesis". En: Muysken \& Smith (eds.), 57-60. 